\title{
A new bioactive diterpenoid from Pestalotiopsis adusta, an endophytic fungus from Clerodendrum canescens
}

\author{
Ming-Feng Xu, Ou-Ya Jia, Sheng-Jia Wang and Qin Zhu* \\ Zhejiang Provincial Key Laboratory for Genetic Improvement and Quality Control of \\ Medicinal Plants, \\ Hangzhou Normal University, Hangzhou 310036, P.R. China
}

*Corresponding author. E-mail: zhuqin@hznu.edu.cn

Bioassay-guided fractionation of the culture extract of Pestalotiopsis adusta, an endophytic fungus isolated from the medicinal plant Clerodendrum canescens, led to the isolation of one new, (10S)-12,16-epoxy-17(15 $\rightarrow 16)$-abeo-3,5,8,12,15-abietapentaen-2,7,11,14-tetraone (1), and four known diterpenoids, teuvincenone F (2), uncinatone (3), coleon U (4), coleon U-12-methyl ether (5). These structures were identified by using spectroscopic methods, including UV, MS, 1D and 2D NMR experiments. This is the first report of these compounds being isolated from a Pestalotiopsis species. The cytotoxic activities of the compounds were evaluated, and compounds $\mathbf{1}$ and $\mathbf{3}$ demonstrated cytotoxic activities against the HL-60 tumor cell line $\left(\mathrm{IC}_{50}<20 \mu \mathrm{M}\right)$.

Keywords: Pestalotiopsis adusta; endophytic fungus; abietane diterpenes; cytotoxic activity 


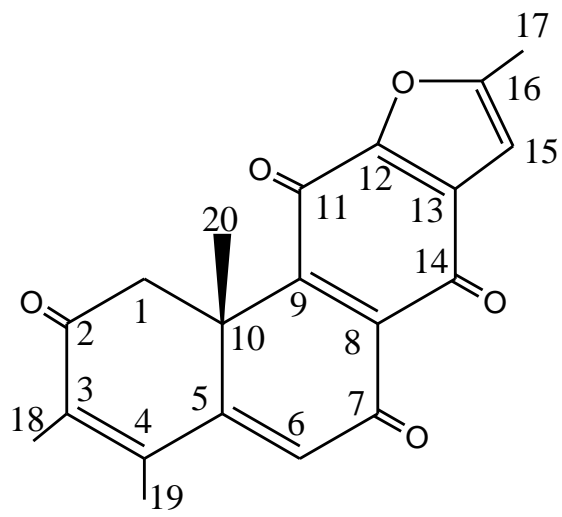

Figure S1 Chemical structure of compound 1

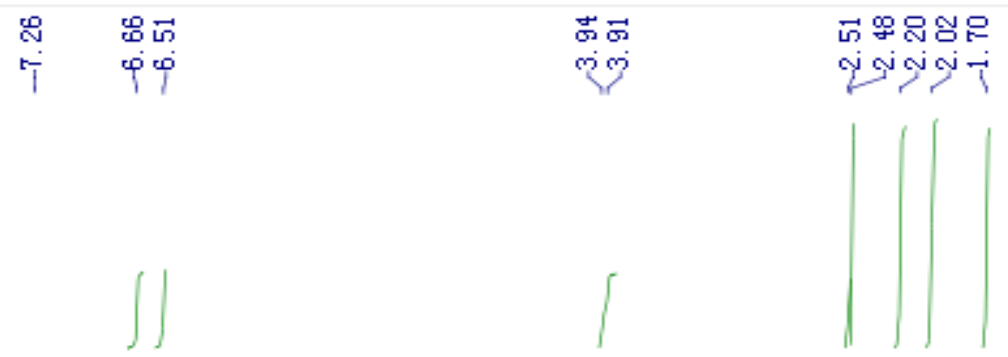

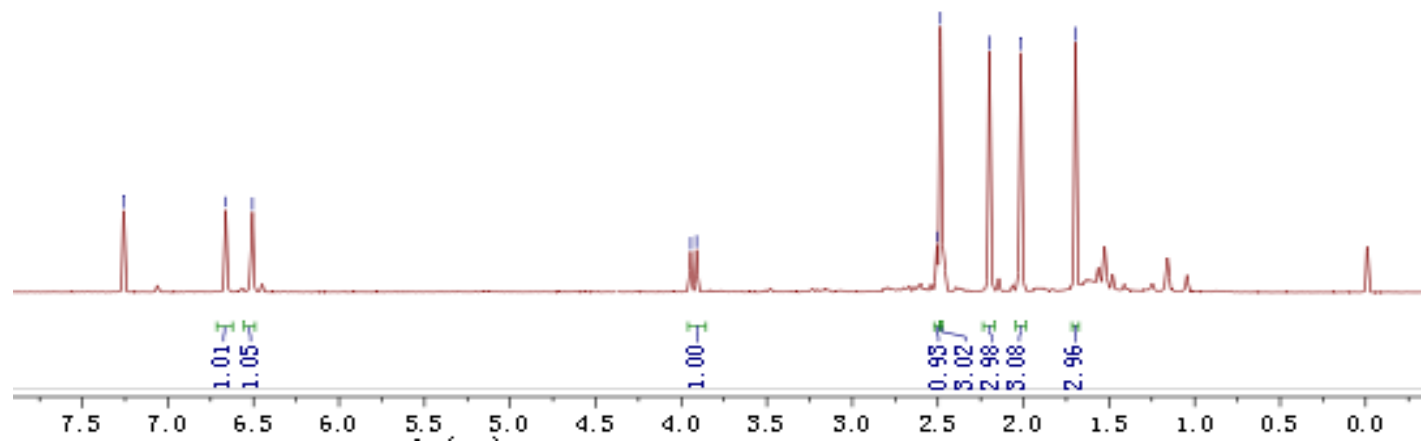

Figure S2. ${ }^{1} \mathrm{H}$ NMR of compound $\mathbf{1}$ 


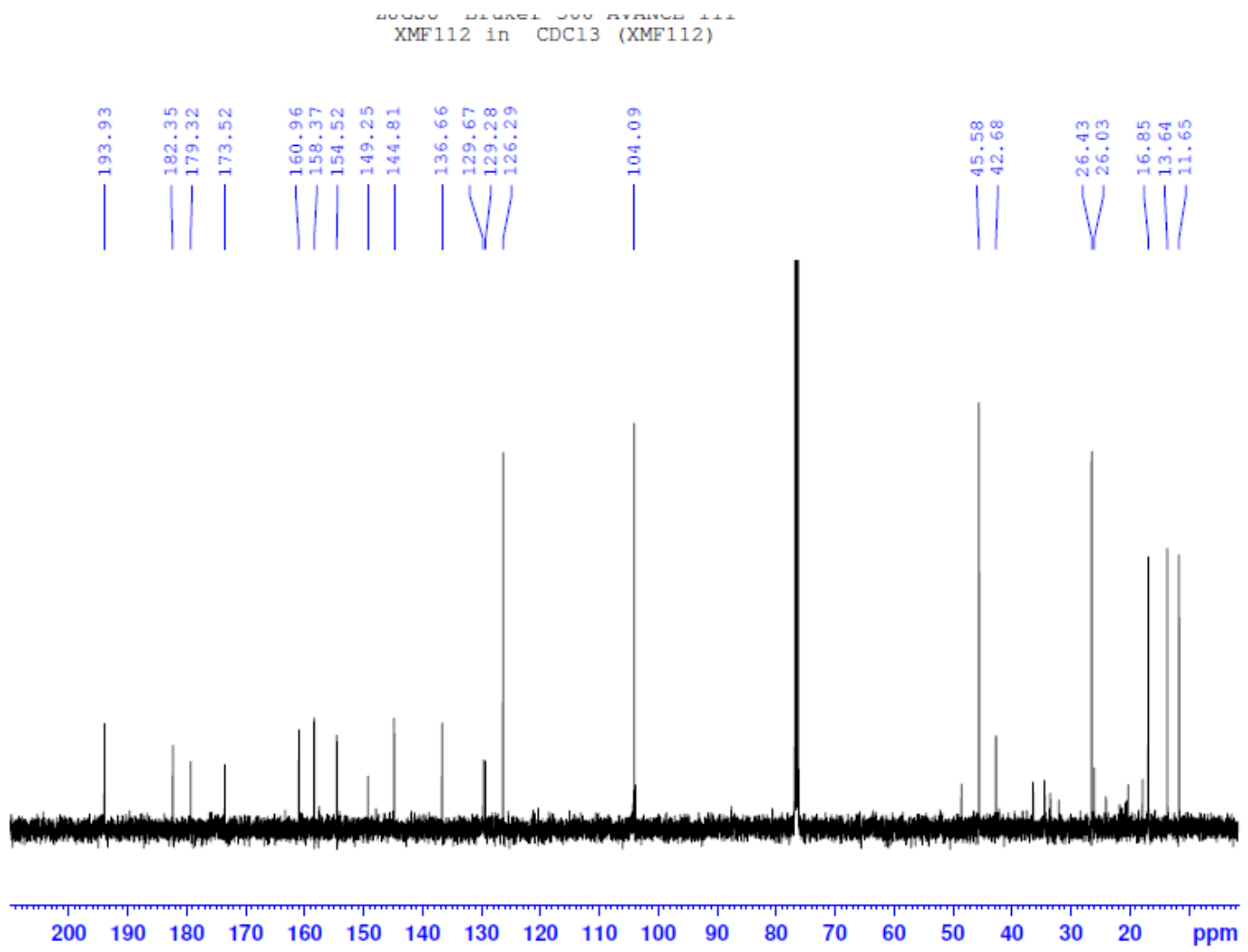

Figure S3. ${ }^{13} \mathrm{C}$ NMR of compound 1

XMF112 in $\operatorname{CDC13}$ (XMF112) DEPT135
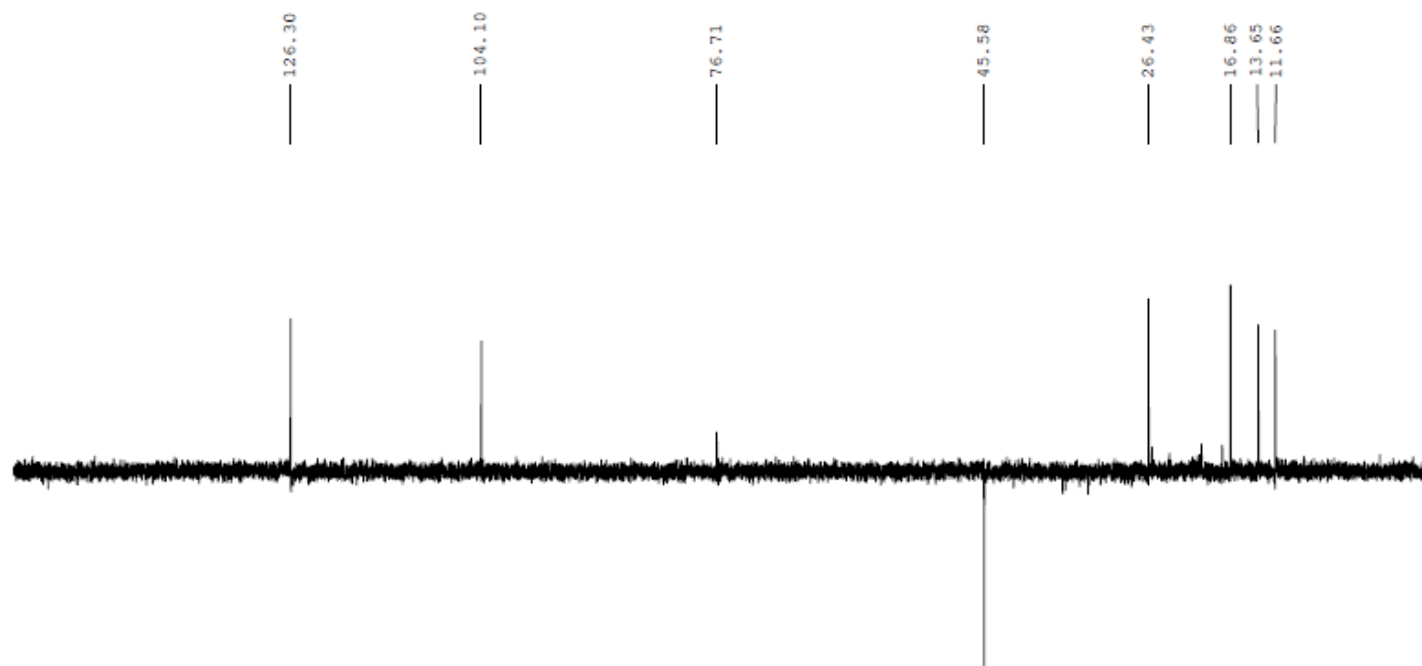

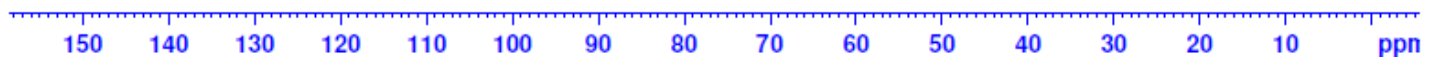

Figure S4. DEPT 135 NMR of compound 1 


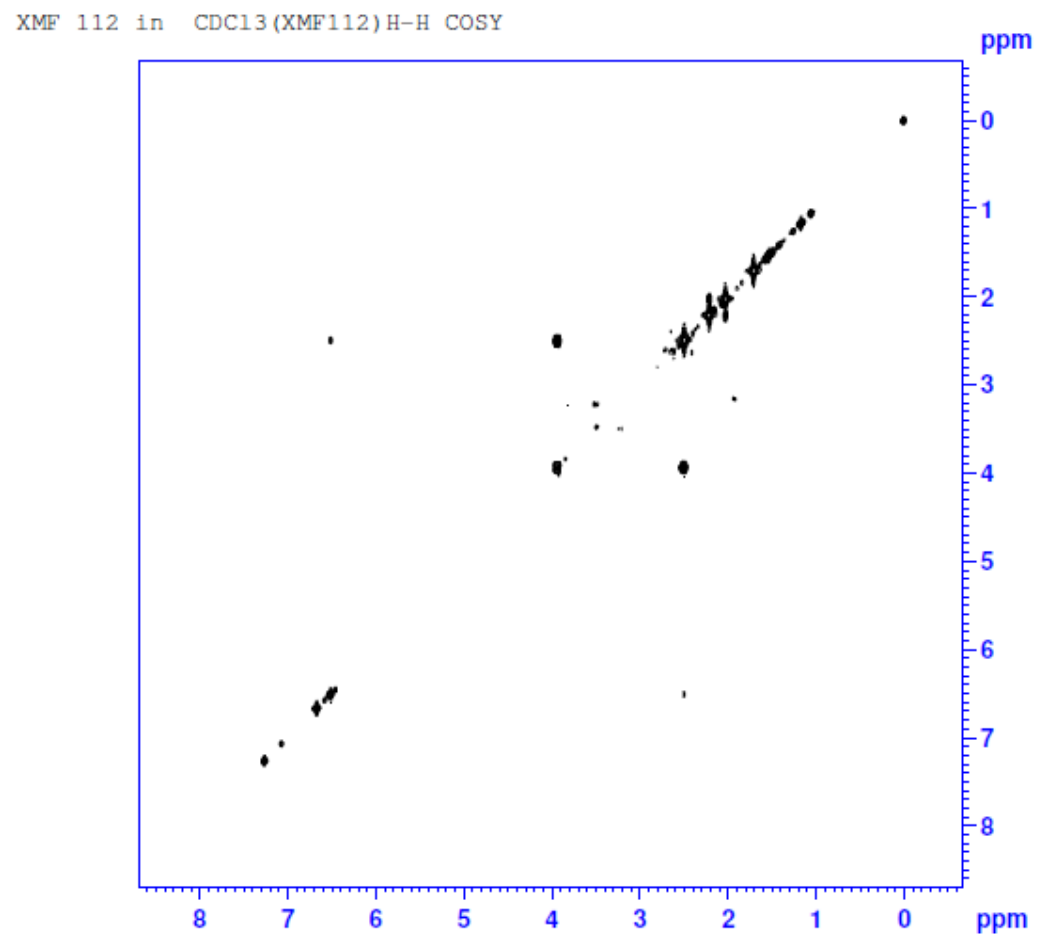

Figure S5. H-H COSY of compound 1

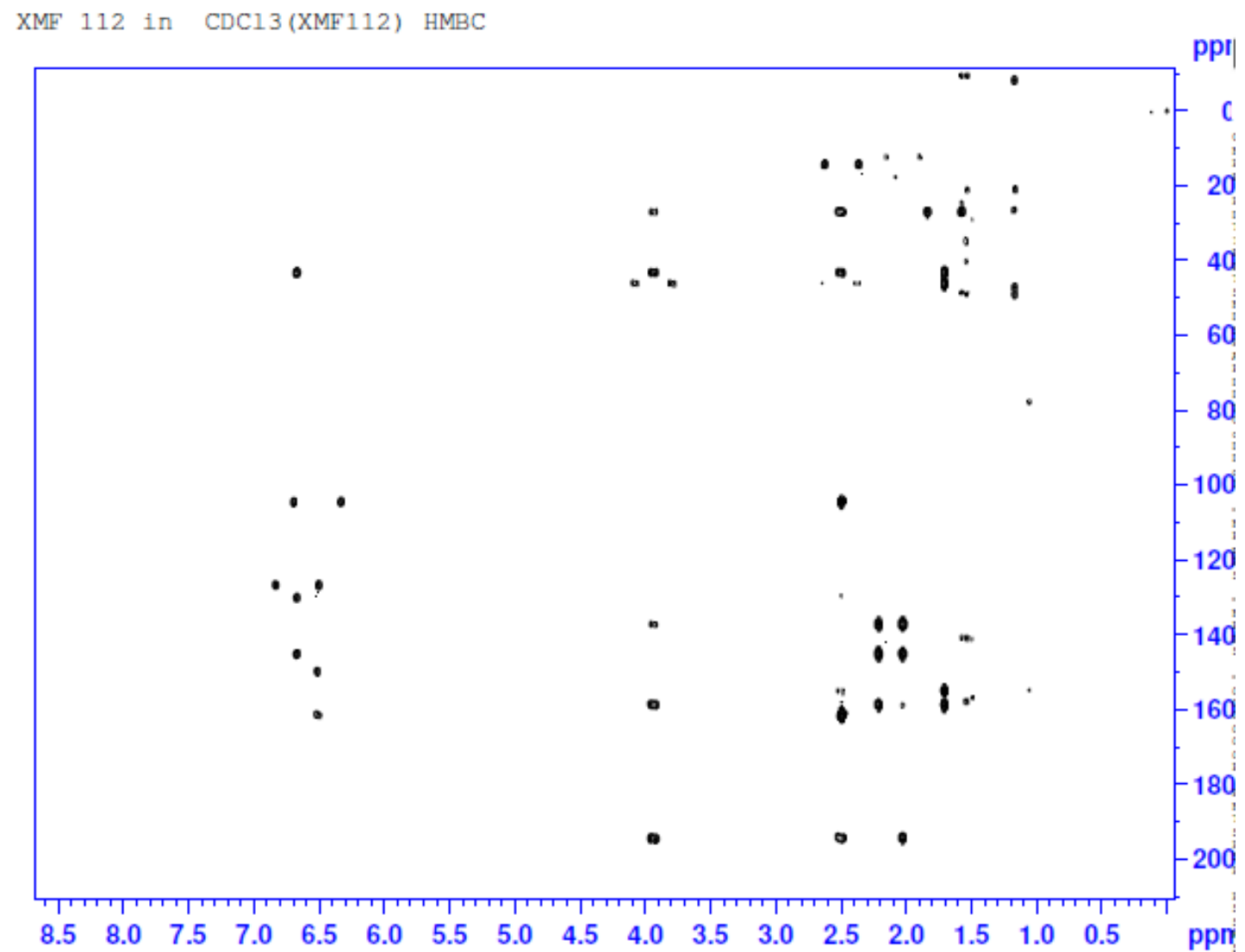

Figure S6. HMBC of compound 1 


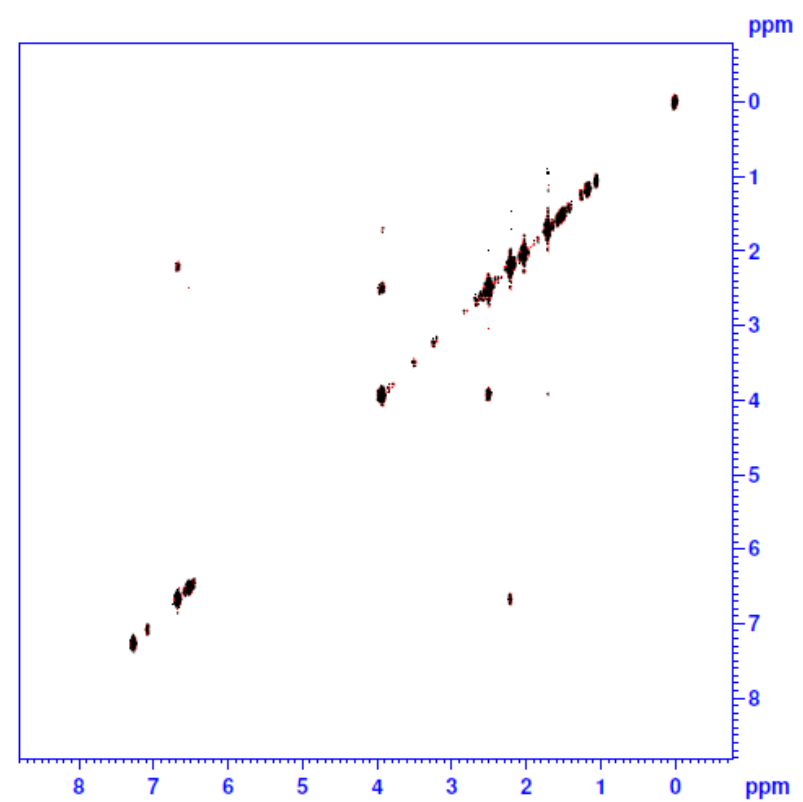

Figure S6. NOESY of compound 1

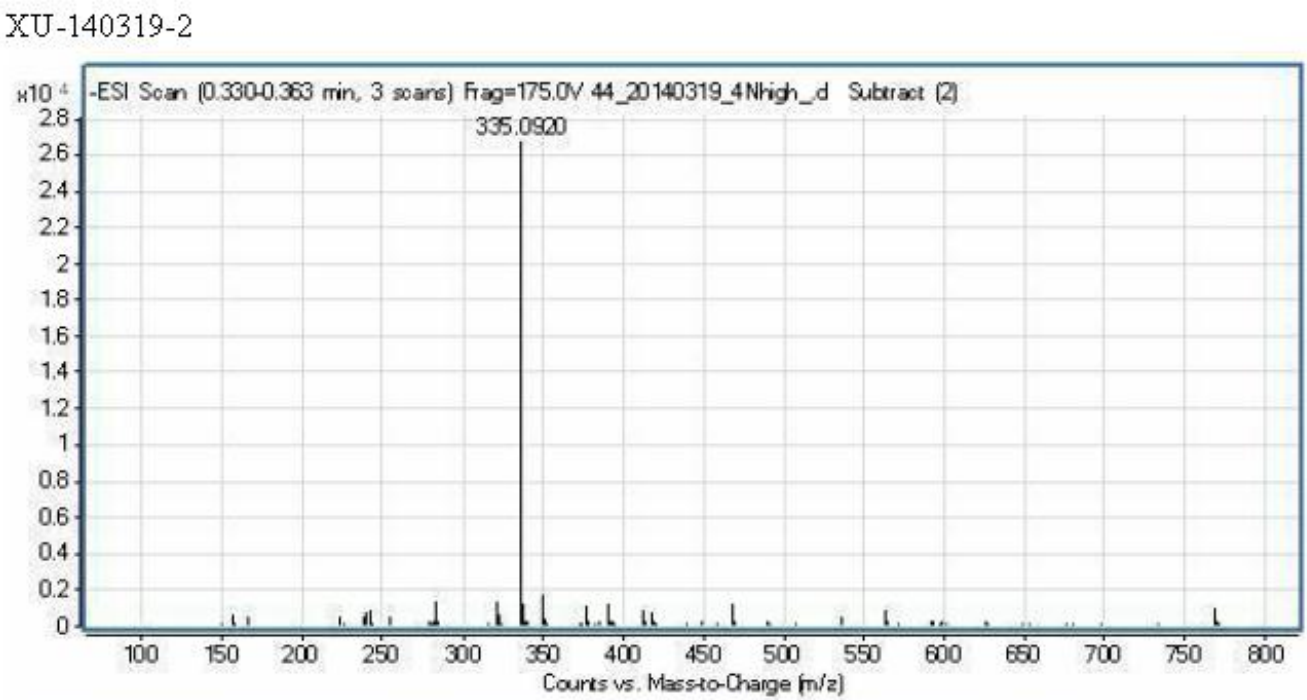

\begin{tabular}{|c|c|c|c|c|}
\hline Ion Formula & $m / z$ & Calc m/z & Diff $(\mathrm{ppm})$ & DEE \\
\hline $\mathrm{C} 20 \mathrm{H} 1505$ & 335.092 & 335.0919 & -0.29 & 13.5 \\
\hline
\end{tabular}

Figure S7. MS of compound 1 


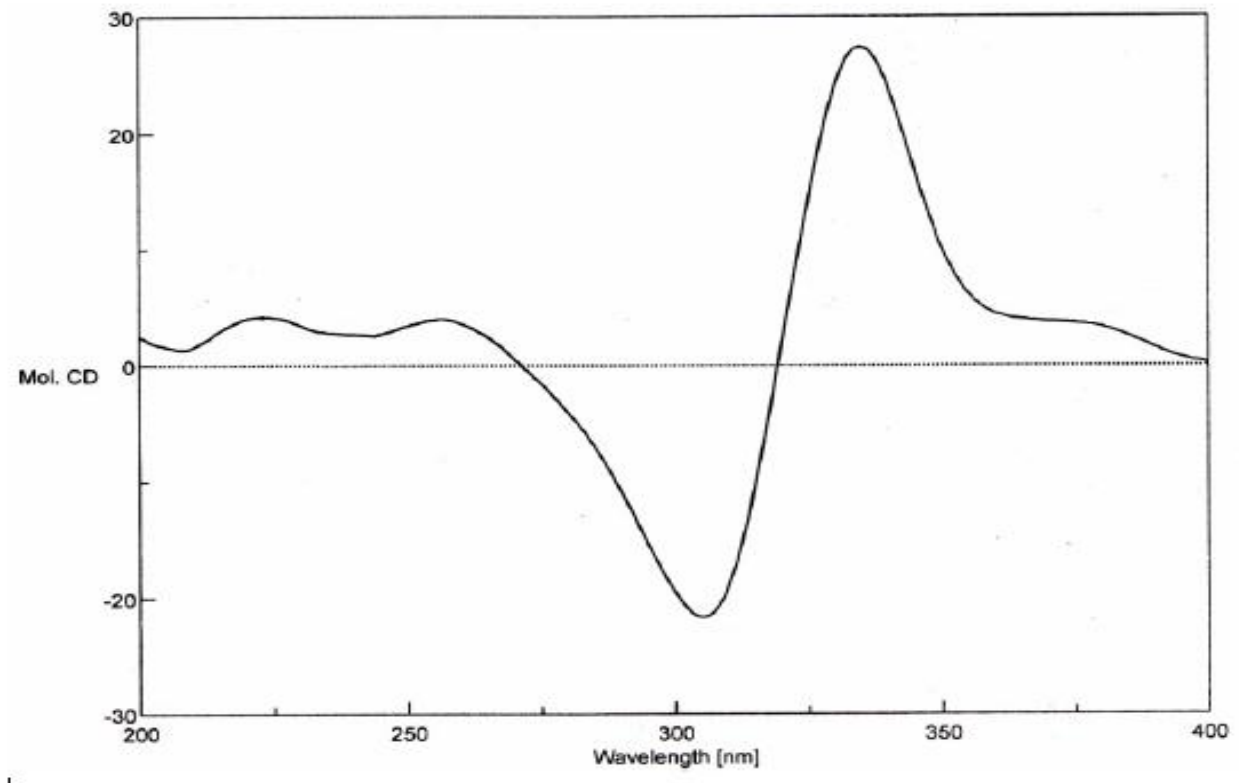

The ECD spectrum of compound $1(\mathrm{MeOH})$

Figure S8. ECD spectrum of compound 1

Table S1 NMR spectroscopic data in $\mathrm{CDCl}_{3}$.for compound $\mathbf{1}$.

\begin{tabular}{|c|c|c|c|}
\hline $\mathrm{H} / \mathrm{C}$ & $\delta_{\mathrm{H}}(J$ in $\mathrm{Hz})$ & $\delta_{\mathrm{C}}$ & HMBC \\
\hline 1 & $2.51, m, \alpha ; 3.91, d d(16.5,9.3) \beta$ & $45.5, \mathrm{t}$ & $\mathrm{C}-2, \mathrm{C}-3, \mathrm{C}-10, \mathrm{C}-20$ \\
\hline 2 & - & $193.9, \mathrm{~s}$ & - \\
\hline 3 & - & $136.6, \mathrm{~s}$ & - \\
\hline 4 & - & $144.8, \mathrm{~s}$ & - \\
\hline 5 & - & $158.3, \mathrm{~s}$ & - \\
\hline 6 & $6.66, s$ & $126.2, \mathrm{~d}$ & C-4, C-8, C-10 \\
\hline 7 & - & $182.3, \mathrm{~s}$ & - \\
\hline 8 & - & 129.6, s & - \\
\hline 9 & - & $154.5, \mathrm{~s}$ & - \\
\hline 10 & - & $42.6, \mathrm{~s}$ & - \\
\hline 11 & - & $179.3, \mathrm{~s}$ & - \\
\hline 12 & - & $160.9, \mathrm{~s}$ & - \\
\hline 13 & - & $129.2, \mathrm{~s}$ & - \\
\hline 14 & - & $173.5, \mathrm{~s}$ & - \\
\hline 15 & $6.51, s$ & 104.0, d & C-12, C-16 \\
\hline 16 & - & $149.2, \mathrm{~s}$ & - \\
\hline 17 & $2.48, s$ & $13.6, \mathrm{q}$ & $\mathrm{C}-15$ \\
\hline 18 & $2.02, s$ & $11.6, \mathrm{q}$ & C-3, C-4, C-5, C-19 \\
\hline 19 & $2.20, s$ & $16.8, \mathrm{q}$ & C-3, C-4, C-5, C-18 \\
\hline 20 & $1.70, s$ & $26.4, \mathrm{q}$ & C- $1, \mathrm{C}-5, \mathrm{C}-10$ \\
\hline
\end{tabular}

${ }^{1} \mathrm{H}$ and ${ }^{13} \mathrm{C}$ NMR data were measured at 500 and $125 \mathrm{MHz}$, respectively. Chemical shifts are given in $\delta$ values.

Abbreviations: s, singlet; m, multiplet; dd, doublet of doublets. 
teuvincenone $\mathrm{F}(2){ }^{13} \mathrm{C}-\mathrm{NMR}\left(125 \mathrm{MHz}, \mathrm{CDCl}_{3}\right) \delta: 46.0(\mathrm{C}-1), 197.4(\mathrm{C}-2), 136.4$ (C-3), 146.2 (C-4), 161.3 (C-5), 123.8 (C-6), 190.3 (C-7), 108.9 (C-8), 131.4 (C-9), 42.6 (C-10), 131.4 (C-11), 152.2 (C-12), 117.7 (C-13), 155.4 (C-14), 101.5 (C-15), 148.8 (C-16), 14.9 (C-17), 12.0 (C-18), 17.5 (C-19), 25.4 (C-20).

uncinatone (3) ${ }^{13} \mathrm{C}-\mathrm{NMR}\left(125 \mathrm{MHz}, \mathrm{CDCl}_{3}\right)$ 8: $29.2(\mathrm{C}-1), 30.2(\mathrm{C}-2), 140.4(\mathrm{C}-3)$, 125.3 (C-4), 165.2 (C-5), 118.5 (C-6), 190.1 (C-7), 109.3 (C-8), 136.1 (C-9), 39.5 (C-10), 130.8 (C-11), 153.7 (C-12), 110.9 (C-13), 154.2 (C-14), 34.5 (C-15), 83.0 (C-16), 22.1 (C-17), 20.6 (C-18), 14.9 (C-19), 22.0 (C-20).

coleon U (4) ${ }^{13} \mathrm{C}-\mathrm{NMR}\left(125 \mathrm{MHz}, \mathrm{CDCl}_{3}\right)$ ): 28.9 (C-1), 18.4 (C-2), 36.0 (C-3), 36.5 (C-4), 141.3 (C-5), 143.2 (C-6), 182.3 (C-7), 104.8 (C-8), 137.4 (C-9), 40.8 (C-10), 132.4 (C-11), 150.3 (C-12), 117.8 (C-13), 156.0 (C-14), 26.2 (C-15), 20.2 (C-16), 20.2 (C-17), 26.6 (C-18), 31.5 (C-19), 27.4 (C-20).

coleon U-12-methyl ether $(\mathbf{5}){ }^{13} \mathrm{C}-\mathrm{NMR}\left(125 \mathrm{MHz}, \mathrm{CDCl}_{3}\right)$ 8: 29.8 (C-1), 17.5 (C-2), 36.3 (C-3), 36.5 (C-4), 138.4 (C-5), 141.9 (C-6), 183.6 (C-7), 109.1 (C-8), 145.5 (C-9), 41.5 (C-10), 135.7 (C-11), 156.1 (C-12), 125.6 (C-13), 151.2 (C-14), 26.0 (C-15), 20.30 (C-16), 20.30 (C-17), 27.0 (C-18), 27.3 (C-19), 27.9 (C-20), 62.1 (C-21). 